\title{
PERFORMANCE SIMULATION AND BER COMPARISION OFDM-MIMO SYSTEMS IN AWGN AND RAYLEIGH FADING CHANNELS WITH BPSK MODULATION SCHEMES
}

\author{
Sandeep Kumar Jain, Lalit Bhanwrela and Pritesh Kumar Jain \\ Department of Electronics and Communication Engineering, Shri Vaishnav Vidyapeeth Vishwavidyalaya, India
}

\begin{abstract}
Behavior of communication medium play vital role in the growth of wireless communication system. To understand various wireless channel environments such as additive white Gaussian noise (AWGN) and Rayleigh fading channels in orthogonal frequency division multiplexing (OFDM) along with multiple input and multiple output (MIMO) system, we analyze mathematical relation of bit error rate (BER) against Signal to Noise ratio (SNR) and verified the same using MATLAB simulation, while considering the BPSK modulation scheme. The performance measured in terms of BER is evaluated for various channels of OFDM-MIMO system along with variation in number of antennas at transmitter and receiver side. It has been demonstrated that the BER and probability of detection improves against SNR per bit, as the diversity employs at transmitter or/and receiver end.
\end{abstract}

Keywords:

Bit Error Rate (BER), Signal to Noise Ratio (SNR), AWGN, Rayleigh Fading Channel, OFDM, MIMO, BPSK

\section{INTRODUCTION}

Recent growth in wireless communication supports new applications with the requirement of high data rates as well as facing problem of fading due to multipath propagation which is considered as one of the major challenge for the current technology. Multipath phenomenon defined as: when multiple radio signals received at the receiver side with different amplitudes, delayed and different phase values. As the demands of higher data rates rapidly increasing day by day for advanced applications of $3 \mathrm{G} / 4 \mathrm{G} / 5 \mathrm{G}$ cellular networks with other wireless services. While designing a wireless communication model, we need to consider the free space channel environment with its effects over the transmitted signal. So we have incorporated the estimation of channel characteristics with respect to the given wireless environment. In this paper, we have studied and analysed the performance of various communication channels named as additive white Gaussian noise (AWGN) and Rayleigh channels along with proposed OFDM-MIMO system model.

In this paper, we have gone through different channels as prerequisite for the data transmission however we have considered the fading environment in case of wireless communication. We emphasized on the AWGN, Rayleigh, OFDM and MIMO channels to estimate their performances based on BER analysis with respect to signal to noise ratio (SNR).

As far as this paper is concern; first we have literature review in section 2. In section 3, system model for OFDM based MIMO channel have been described. Then section 4, contains mathematical expression of BER for AWGN, Rayleigh and OFDM based MIMO channels. Simulation results have been described under section 5 and section 6 presents the conclusion.

\section{LITERATURE REVIEW}

During literature review, we observed that as signal to noise/ interference ratio is increased; it will provide improvement in the management and maintenance of wireless network [1] that meets the requirement for the next generation technologies. In addition to the same, some authors [2] [3] have been researched on the evaluation to the OFDM based MIMO channel where BER and SNR performance analysed over various wireless channel. In one of the paper [4], author presented research on analysis of BER of multi-processing gain which is carried out for spreading sequence with respect to AWGN and slow Rayleigh fading channels. In paper [5], author presents performance evaluation of BER for a massive MIMO system with M-ary PSK scheme over fading channel.

In the research study we found that following factors are responsible for multipath and investigated as reflection and refraction from ionosphere, ducting in environmental space, scattering due to physical structures of surroundings [6] [7]. We have considered both constructive as well as destructive case of interferences under the multipath phenomenon [8]. Additional issues such as spectrum scarcity and interference with the presence of other mobile users, this obligation calls for the improvement in the wireless connections with higher spectral efficiency. In order to improve system performance while considering all such challenges, we approached with Orthogonal Frequency Division Multiplexing (OFDM) as multi-carrier modulation technique for Multiple Input Multiple Output (MIMO) model to achieve high data rates and reduction in Intersymbol interference (ISI) [9] [10].

\section{OFDM-MIMO SYSTEM MODEL}

As shown in Fig.1, during transmitting stage, serial input data symbols are converted into parallel data symbols and loaded with respective subcarrier. Using IFFT these symbols are converted into samples. To avoid ISI problem, cyclic prefix are added, due to this process, circular convolution get change into linear operation at receiver side, when we perform FFT. After adding cyclic prefix, OFDM blocks are going to be transmitted through Multiple Input and Multiple Output circumference through various wireless channels as AWGN and Rayleigh channel. At the receiving side, reverse process has been carried to estimate input sequence. 


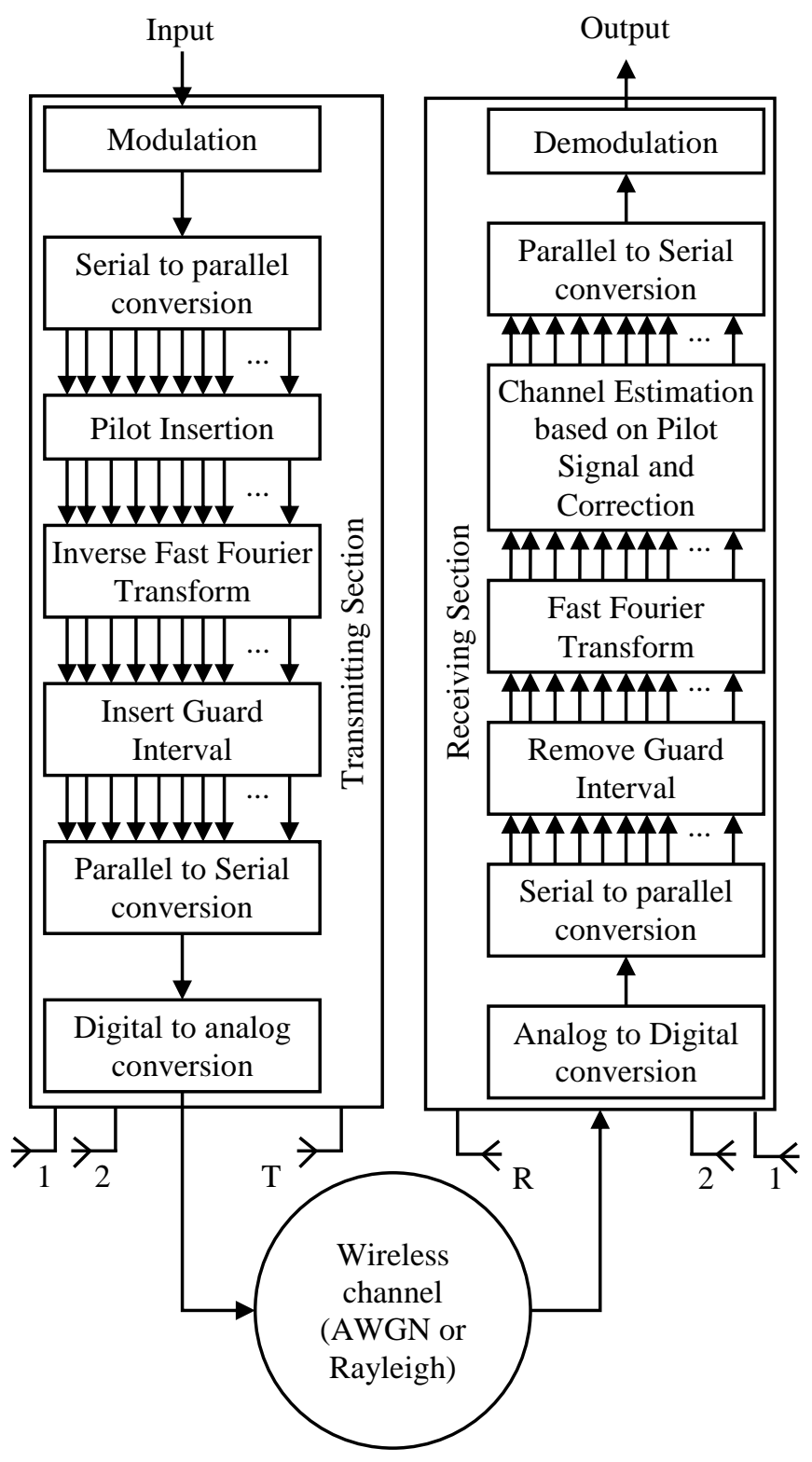

Fig.1. OFDM-MIMO System Model

\section{MATHEMATICAL REPRESENTATION OF VARIOUS CHANNELS}

In this section, we have represented mathematical expression of BER for various channels [11].

\subsection{AWGN CHANNEL}

It defines the basic wire line communication system with noise to illustrate the following characteristics of the thermal noiseadditive noise means that the received signal defined by addition of transmitted signal and noise in such way that noise independent of transmitted signal. White noise exhibits the flat response of PSD. In wired mode of communication AWGN considered for modeling the channel as it is only impairment for the transmitted signal. According to the wire line communication model as shown below for the received signal $o(k)$ as,

$$
o(k)=i(k)+n(k)
$$

where, $i(k)$ is the signal to be transmitted and $n(k)$ specifies the white Gaussian noise.

If transmitted signal is real then real part of the AWGN added to the signal and if the transmitted signal is complex then complex AWGN added that provides complex signal for the received side. BER for AWGN channel can be expressed as:

$$
P\left(n(k)>+\sqrt{P_{S}}\right)=Q\left(\sqrt{\frac{P_{S}}{\sigma_{n}^{2}}}\right)=Q(\sqrt{S N R})
$$

where,

$$
Q(x)=\frac{1}{\sqrt{2 \pi}} \int_{x}^{\infty} e^{\left(\frac{-t^{2}}{2}\right)} d t
$$

and

$$
\operatorname{SNR}(d B)=10 \log _{10}\left(\sqrt{P_{S}}\right)
$$

\subsection{RAYLEIGH CHANNEL}

In real world of wireless communication system by understanding Rayleigh channel, we can define the fading environment for the multipath propagation of the transmitted signal. Factors such as scattering, dispersion, Doppler effects that occurs during the signal transmission and reception have been studied under the fading issues in the wireless communication system. We considered that due to the fading signal magnitude reduced as per the Rayleigh distribution in the multipath propagation environment.

Rayleigh channel output can be characterized as:

$$
o(k)=h_{i}(k)+n(k)
$$

where,

$$
h=e^{j \theta}=\sum_{k=0}^{L-1}\left(i_{k}+\mathrm{j} o_{k}\right)=i+\mathrm{j} o
$$

BER for Rayleigh fading channel under normal SNR can be given as:

$$
\operatorname{BER}(a)=Q\left(\sqrt{\frac{a^{2} P_{S}}{\sigma_{n}^{2}}}\right)=\frac{1}{\sqrt{2 \pi}} \int_{a \sqrt{u}}^{\infty} e^{\left(\frac{-t^{2}}{2}\right)} d t
$$

At high SNR value:

$$
B E R_{\text {Rayleigh }}=0.5\left(1-\frac{\sqrt{\frac{\mu}{2}}}{\sqrt{1+\frac{\mu}{2}}}\right)=0.5\left(1-\sqrt{\frac{S N R}{2+S N R}}\right)
$$

where,

$$
\mu=\frac{P_{S}}{\sigma_{n}^{2}}
$$

\subsection{ORTHOGONAL FREQUENCY DIVISION MULTIPLEXING (OFDM)}

OFDM technique is the combination of multi-carrier modulation and multiplexing to achieve higher data rates as well as spectral efficiency. This technology bears the challenges of wireless communication and finds applications in various advanced wireless services. Using OFDM we divided a wideband 
into several frequency components known as subcarriers, which carries the data. Further we transmitted various subcarriers simultaneously without any interference as they are orthogonal to each other. BER calculation for OFDM channel is given as,

$$
\begin{gathered}
O(k)=H(k) I(k)+N(k) \\
N(k)=\sum_{m=0}^{N-1} n(m) \exp \left(-j 2 \pi \frac{k m}{N}\right) \\
\sigma_{N}^{2}=E\left\{|N(k)|^{2}\right\}=N \sigma_{N}^{2} \\
H(k)=\sum_{m=0}^{N-1} h(m) \exp \left(-j 2 \pi \frac{k m}{N}\right) \\
E\left\{|H(k)|^{2}\right\}=L \\
A v g \_S N R=\frac{L P}{N \sigma_{N}^{2}} \\
B E R_{\text {OFDM }}=0.5\left(1-\sqrt{\frac{L P}{N \sigma_{N}^{2}}} \frac{L P}{2+\frac{L \sigma_{N}^{2}}{2}}\right.
\end{gathered}
$$

\subsection{MULTIPLE INPUT MULTIPLE OUTPUT (MIMO)}

MIMO considered as one of the technology which fulfill the requirements of higher spectral efficiency, through gain of spatial multiplexing and reliable connections, through diversity achieved by multiple antennas.

\subsubsection{Model for MIMO:}

Let number of transmitter and receiver antennas are $T$ and $R$ respectively. For MIMO channel, the received signal $o$ can be written as,

$$
o=H^{*} i+n
$$

where,

$$
\begin{gathered}
o=\left[\begin{array}{c}
o_{1} \\
o_{2} \\
o_{3} \\
\vdots \\
o_{R}
\end{array}\right] \\
i=\left[\begin{array}{c}
i_{1} \\
i_{2} \\
i_{3} \\
\vdots \\
i_{T}
\end{array}\right] \\
H=\left[\begin{array}{ccc}
h_{11} & \cdots & h_{1 T} \\
\vdots & \ddots & \vdots \\
h_{R 1} & \cdots & h_{R T}
\end{array}\right]
\end{gathered}
$$

\section{SIMULATION RESULT}

Comparison among various channels have been simulated in Fig.2, Which shown relation between BER and SNR, with respect to increasing the SNR corresponding BER value has been decreased in all channels. Due to absence of multipath and fading effect, AWGN provides best SNR characteristic. The Fig.3 will provide the verification result of MIMO channel in case of transmit and receive antenna are same as 2 .

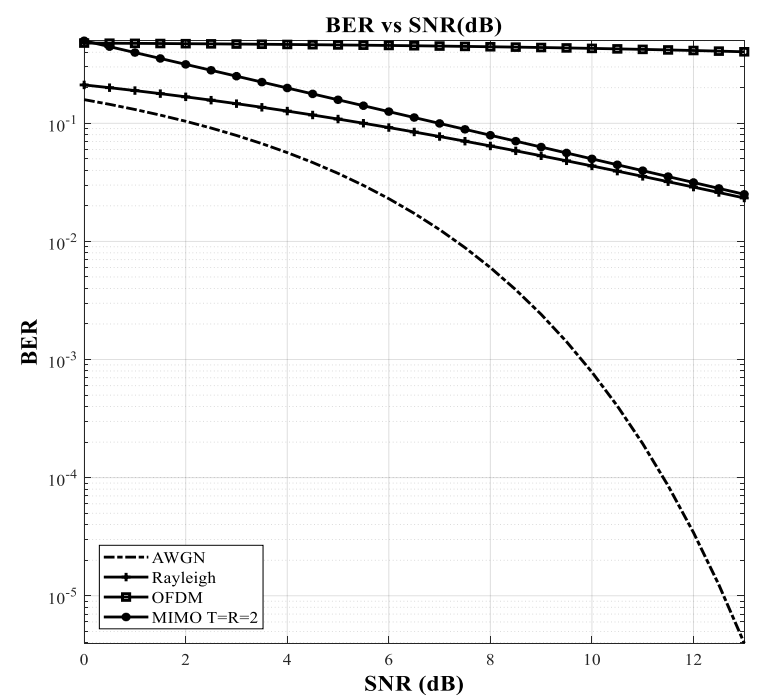

Fig.2. Comparison among various wireless environments

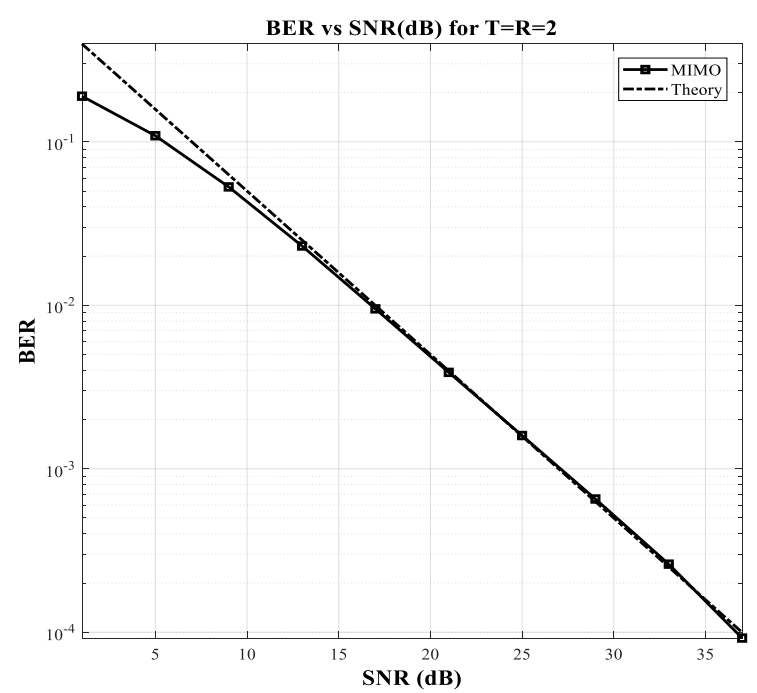

Fig.3. MIMO Channel for $T=R=2$

As we employ diversity at receiver end, while increasing number of antennas, BER value significantly decreased. For example, here in Fig.4, it is clear that for a fixed value of $25 \mathrm{~dB}$ SNR, BER value changes from approximate $10^{-3}$ to $10^{-10}$ as number of antennas changed from $R=2$ to $R=5$. 


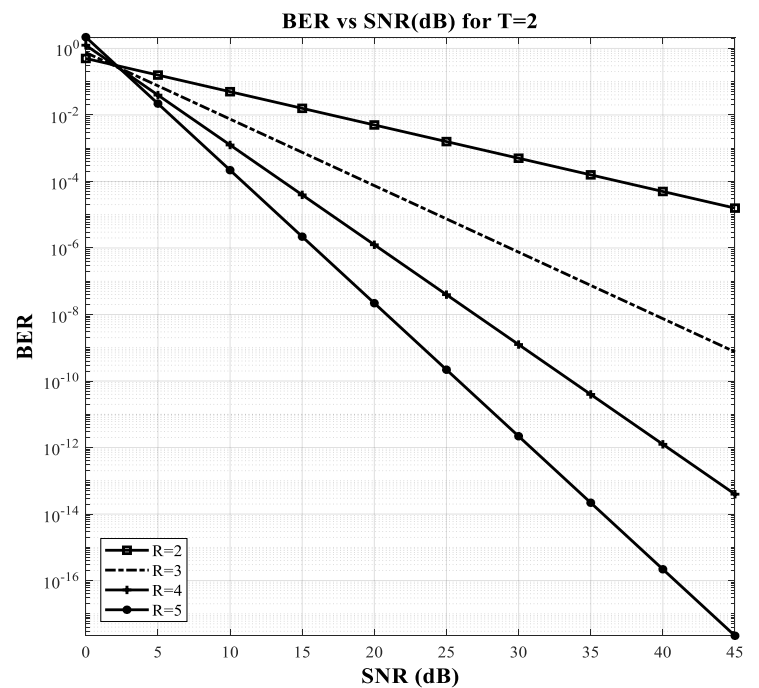

Fig.4. MIMO with variable number of antennas at receiver

In Fig.5, we represent relation among probability of detection with SNR for different value of antennas at receiver end while keeping number of transmitter antenna fixed as 2. Here also we can observe that as diversity employs, probability of detection gets better. Let's consider the fixed value of sensing SNR is $5 \mathrm{~dB}$, we gets much better detection if $R=5$ in comparison of $R=2,3$, 4 , which shows diversity plays significant role to provide accuracy in the process of detection.

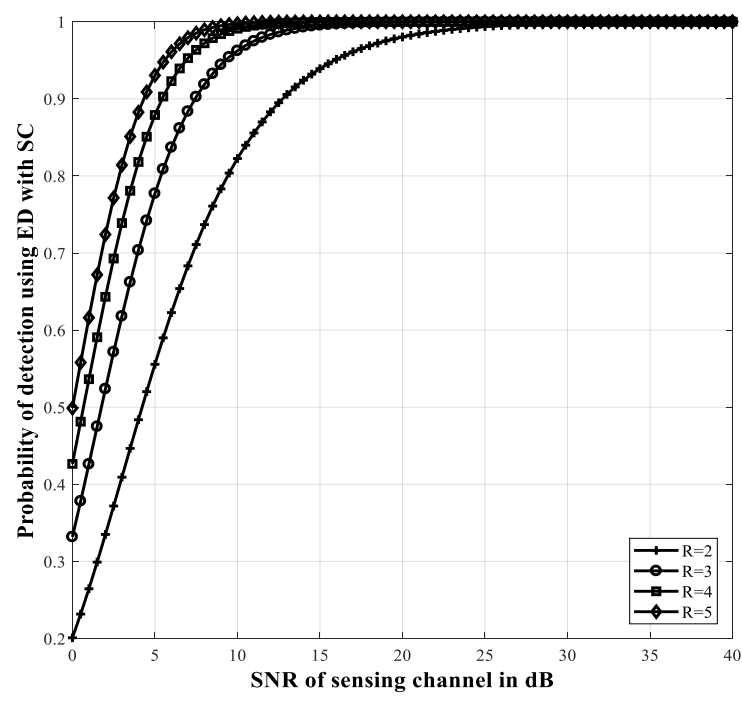

Fig.5. Probability of Detection

\section{CONCLUSIONS}

Performance of wireless communication system depend on various factors, one of them is behavior of communication medium which play vital role. In this paper we provide comparative study among various wireless channel environments such as additive white Gaussian noise (AWGN) and Rayleigh fading channels in orthogonal frequency division multiplexing (OFDM) along with multiple input and multiple output (MIMO) system.
While taking BPSK modulation scheme in consideration, we have also analyzed mathematical relation of bit error rate (BER) against Signal to Noise ratio (SNR) and verified the same using MATLAB simulation. In order to understand effect of diversity on BER and probability of detection for various channels of OFDM-MIMO system, number of antennas at transmitter and receiver side has been varied. When we employ diversity, it has been demonstrated that the BER and probability of detection improves against SNR.

\section{REFERENCES}

[1] M. Milosavljevic, S. Sofianos, P. Kourtessis and M. John, "Self-Organized Cooperative 5G RANs with Intelligent Optical Backhauls for Mobile Cloud Computing", Proceedings of IEEE Workshop on Optical-Wireless Integrated Technology for Systems and Networks, pp. 900904, 2013.

[2] A. Kammoun, H. Khanfir, Z. Altman, M. Debbah and M. Kamoun, "Preliminary Results on 3D Channel Modeling: From Theory to Standardization", IEEE Journal on Selected Areas in Communications, Vol. 32, No. 6, pp. 1219-1229, 2014.

[3] J. Koppenborg, H. Halbauer, S. Saur and C. Hoek, "3D Beamforming Trials with an Active Antenna Array", Proceedings of ITG Workshop on Smart Antennas, pp. 2124, 2012.

[4] Selim Dilek and Oguz Kucur, "Performance of MultiProcessing Gain QS-CDMA over AWGN and Rayleigh Fading Channels", Proceedings of International Conference on Signal Processing and Communications Application, Vol. 20, pp. 1207-1214, 2010.

[5] Joy Iong Zong Chen, Bo Hueng Lee and Wen Bin Wu, "Performance Evaluation of BER for an Massive-MIMO with M-ary PSK Scheme over Three-Dimension Correlated Channel", Computer and Electrical Engineering, Vol. 65, pp. 196-206, 2018.

[6] Theodore S.Rappaport, "Wireless Communication Principles and Practice", 2 ${ }^{\text {nd }}$ Edition, Pearson Education Press, 2010.

[7] John G. Proakis, "Digital Communications", $4^{\text {th }}$ Edition, McGraw-Hill, 2001.

[8] B. Sklar, "Rayleigh Fading Channels in Mobile Digital Communication Systems Part II: Mitigation", IEEE Communications Magazine, Vol. 35, No. 9, pp. 148-155, 1997.

[9] Sinem Coleri, Mustafa Ergen, Anuj Puri and Ahmad Bahai, "A Study of Channel Estimation in OFDM Systems", Proceedings of IEEE $56^{\text {th }}$ Vehicular Technology Conference, pp. 894-898, 2002.

[10] Xiaojuan Chen, Minglei Zhang and Xue Han, "Performance Analysis of Reception for OFDM Communication Systems", Proceedings of International Conference of Information Technology, Computer Engineering and Management Sciences, pp. 732-736, 2011.

[11] Aditya K. Jagannatham, "Principle of Modern Wireless Communication Systems Theory and Practice", McGraw Hill Education, 2016. 